\title{
Use of 3D Body Scanner Data in Digital Tailoring
}

\author{
Pirjo ELBRECHT*a,b \\ a Tallinn University of Technology, Tallinn, Estonia; \\ ${ }^{\mathrm{b}}$ Incognito Ballistic, Tallinn, Estonia \\ http://dx.doi.org/10.15221/13.076
}

\begin{abstract}
Precise measurements are the key for making digitally tailored garments. Therefore it is necessary to have measurement defined by the measurement extraction profile which would correspond to apparel basic block construction. A block is a foundation pattern constructed to fit a specific human. A block should be defined as formulas to the CAD/CAM system (Gemini) or altered by CAD/CAM systems (Lectra, Optitec, Gerber, etc) to fit an individual figure using personal measurements.

The data captured by body scanner can be very precise depending on the technology (white-light, infrared depth sensor, etc) and the amount of detectors (lasers/sensors/lights) used. The precision of measurements obtained depends on how exactly the body scanner detects the body surface and how good is the measurement extraction profile. At the moment, there can happen that even two different versions of scanners from the same company can produce results with $3-4 \mathrm{~cm}$ difference in knee circumference (depending on the knee circumference).

After considering over 10000 body scans, I have come to conclusion, that the existing scanners do not detect well enough crotch area (the region of the body where the legs join the torso, and are often considered to include the groin and genitals). Measurement extraction profiles do not allow to map crotch point (the crotch point is the point starting from which the cross-section of the body changes from one circle to two circles) as it is constructed in the persons block where the legs join together and the bottom of the crotch defines one end of the inseam.

We should have a standardized "human" prototype as a tool to compare results produced by different scanners in order to secure the precision of the body scanner data and measurement extraction profiles.
\end{abstract}

Keywords: Digital tailoring, 3D scanners, body scanning, landmarks, measurements extraction, computer-aided anthropometry, crotch point, CAD/CAM system, basic block, standardized "human" prototype

\section{Introduction}

Patterns for tailor made garments are drafted by skilled pattern makers according to what they have measured and observed. This task requires considerable expertise and experience and thus induces great labor costs. With the development of CAD/CAM technologies, the computer software can generate clothing patterns more efficiently [1], and based on the mathematical expressions for required measures, the computerized construction of clothing patterns can be realized [2]. Besides, the computer-generated human models help to visualize the fitting results of the designed clothes in virtual environment [3] and [4] due to the consistency of computer programs, patterns are created according to the same rules repeatedly. Moreover, the data can be stored and retrieved with ease. It has the flexibility of generating a different type of clothing pattern rapidly without taking the body measures again.

Digitally tailored garments are designed, created, altered and prepared for the factory digitally according to the customer body dimensions taken by a body scanner, $2 \mathrm{D}$ digital camera, or direct input, so that unique garments can be produced with mass production efficiency. The process is fully automated starting with placing the customer order into a digital tailoring system and ending with cutting machine cutting the fabric into pattern pieces automatically (see Fig. 1. Digital tailoring chain). The process does not require any human intervention, thus reduces labor costs and processing time. The digital tailoring system improves not only customers' satisfaction but also the manufacturers' profits

The integration tool allows the body measurements to be analyzed and sent to CAD systems. The integration tool is based on background knowledge which experienced pattern makers have. The integration tool is necessary to combine the customer Co-Design options and measurements. The system has built-in "tailor knowledge", which is the key element for creating fitting Made-to-Measure products. 
In this study, I will describe problems with body scanner measurement extraction (Tc2), the importance of how measurement extraction profile correspondents to the basic block, and lack of standards to control the precision of the body scanner. These problems are disturbing the development of digital tailoring systems, and are causing inaccuracy in the measurements.

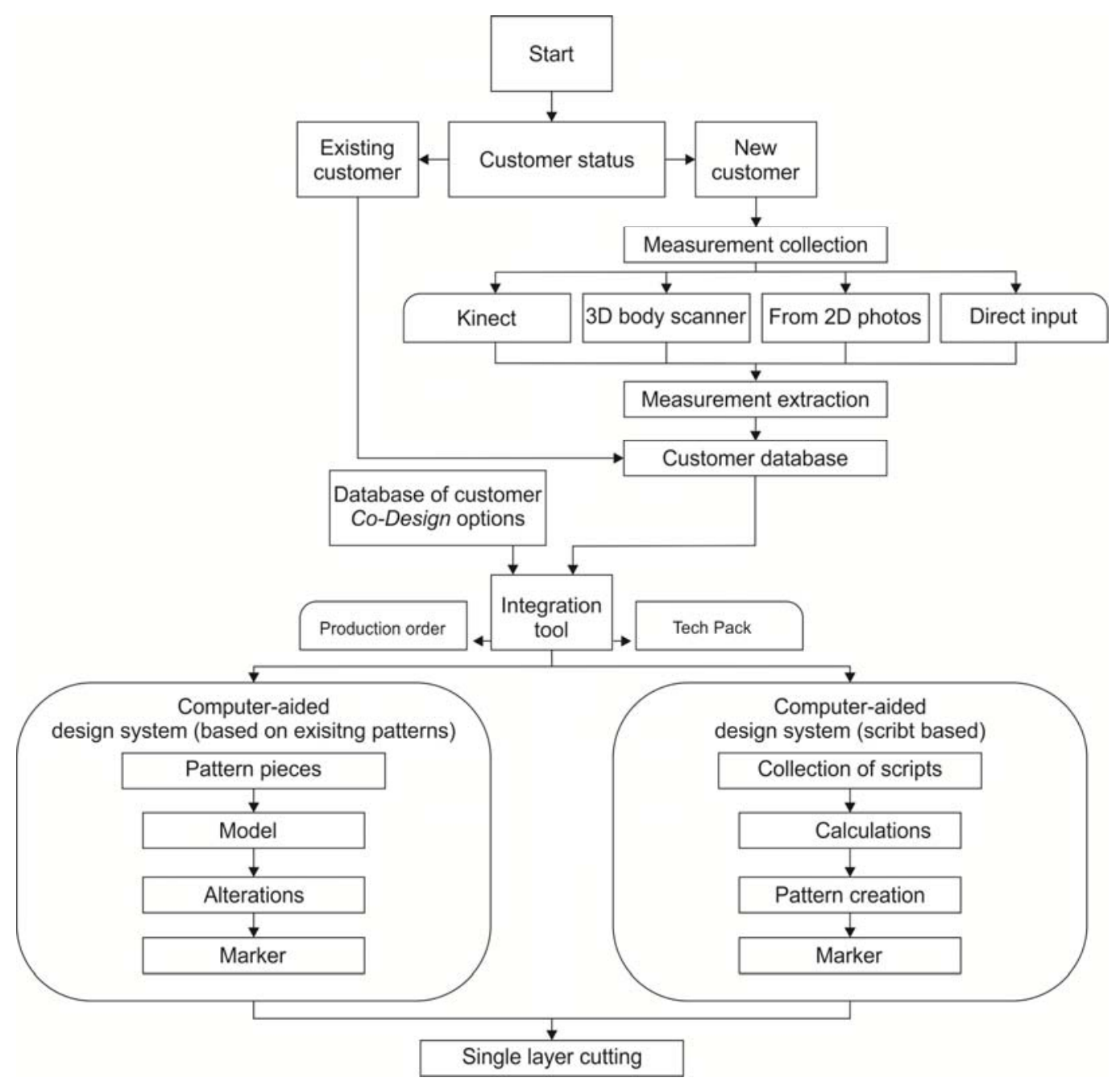

Fig. 1. Digital tailoring chain

\section{Body scanner}

A 3D body scanner is a useful tool that effectively and accurately analyzes the human body and its shape. Once a scan is taken, the produced data will be sent to a computer and visualized on the screen. The output of whole body scanners is a cloud of points, usually transformed in a triangulated mesh through the use of specific algorithms in order to support the 3D visualization of the surface and the extraction of meaningful anthropometric landmarks and measurements.

In the next step, software automatically locates body landmarks and generates measurements. Once a person's body is captured using a body scanner, it can be retrieved, and any part of his/her body can be measured any number of times without actual contact with the measured person. Scan data can be saved in a special format to be read by CAD system or integration tool that allows the body measurements to be analyzed and directed into the CAD system.

\subsection{Problems in everyday work}

I have worked with body scans almost three years - every day. In these three years, I have noticed that the quality of scans has gone down. One of the reasons might be that there is demand for a low cost body scanner and there has not carried enough research before launching the product 


\subsubsection{Body measurements are dependent on the scanner type used}

The body scanner precision needs to be controlled more. There are lots of researches which are comparing body scanner measurements against hand measuring, but none where measurements produced by different scanners would be compared.

In this study, two $\mathrm{Tc}^{2}$ scanners are compared:

- $\quad \mathrm{NX16}$ - on the market from 2007

- KX16 - on the market from 2012

The test group contained 20 people. The whole body of each participant was first scanned with the NX16 and then in the same day with the KX16 in order to compare lower body measurements from seat to calves. Before the measurement extraction was made, all the scans were inspected visually and crotch point position was changed if it was not in required place.

The biggest variation in two different scanner measurements appeared on knees $(7,63 \%$, ca $3 \mathrm{~cm})$ and calves $(6,9 \% \mathrm{ca} 3 \mathrm{~cm})$. If these results are transferred to ready-to-wear clothing sizes this creates difference in two - three sizes (based on Finnish national survey [5]).

The results show that all participants had left side always bigger than the right side (average difference was $2.46 \%$ ) with KX16. In the same time with NX16 body scanner sides, were rather the same or left side was slightly smaller.

The average difference in lower body measurements from two scanners is $2.1 \%$. We obtained similar results when we compared two other NX16 and KX16 body scanners.

The results are shown in table 1 . All the percentages which are bigger than $+/-3 \%$ are highlighted.

From this follows, that if a person is scanned with the NX16 body scanner, he or she would get tighter fitting clothing than with the KX16 body scanner.

Question is, which scanner is more precise? Can we really say that body scanner is detecting measurements precisely and we can produce fitting clothing by using them?

\subsubsection{Corrupt measurements due to the twisted body sides}

The first issue is connected with measurement extraction profile not extracting the body sides in proper way, thus majority of the measurements are erroneous. Example: the right outseam was found on the half way on the right front side and the left outseam was found on the half way on the left back side, also the inseam was corrupt ( Fig. 2 Corrupt measurements)
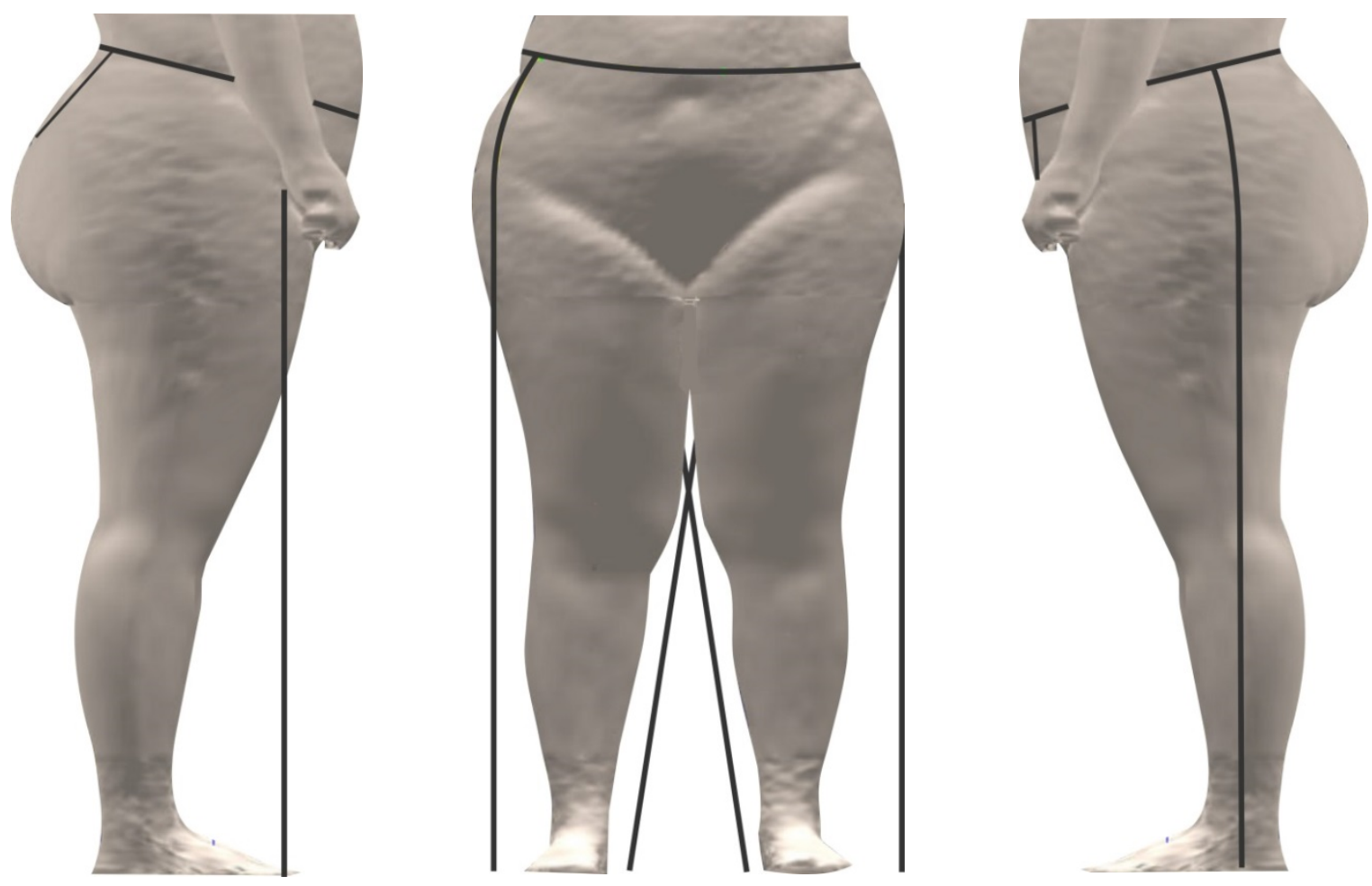

Fig. 2 Corrupt measurements

When this problem was noticed, the customer was rescanned with underwear placement straightened (modified) or underwear changed. This problem does not occur with version NX16. 
Table 1. Body scanner measurement comparison between KX16 and NX16

\begin{tabular}{|c|c|c|c|c|}
\hline Measurement & $\begin{array}{c}\text { Average } \\
\text { difference \% } \\
\text { (KX16-NX16) }\end{array}$ & $\begin{array}{c}\text { MAX } \\
\text { difference \% } \\
\text { (KX16-NX16) }\end{array}$ & $\begin{array}{c}\text { MIN } \\
\text { difference \% } \\
\text { (KX16-NX16) } \\
\end{array}$ & $\begin{array}{c}\text { Fluctuation } \\
\text { between min } \\
\text { max \% } \\
(\text { KX16-NX16) }\end{array}$ \\
\hline Hips_Full & 0,99 & 2,63 & $-1,36$ & 4,00 \\
\hline Hips_Width & 0,56 & 2,53 & $-2,46$ & 4,99 \\
\hline Seat_Full & 1,12 & 1,90 & 0,00 & 1,90 \\
\hline Seat_FrontX & $-0,14$ & 0,65 & $-1,24$ & 1,89 \\
\hline Seat_BackX & $-0,75$ & 0,35 & $-2,40$ & 2,75 \\
\hline Seat_Width & 0,68 & 1,64 & $-0,74$ & 2,37 \\
\hline Knee_Left & 5,06 & 7,63 & 3,73 & 3,90 \\
\hline Knee_Right & 1,97 & 5,46 & 0,71 & 4,76 \\
\hline Knee_Height_Left & 0,45 & 3,94 & $-1,19$ & 5,13 \\
\hline Knee_Height_Right & 0,33 & 3,94 & $-1,49$ & 5,43 \\
\hline UnderKnee_Left & 4,76 & 6,10 & 4,04 & 2,06 \\
\hline UnderKnee_Right & 1,47 & 2,23 & 0,61 & 1,63 \\
\hline UnderKneeHeight_Left & 0,22 & 1,52 & $-1,93$ & 3,45 \\
\hline UnderKneeHeight_Right & 0,92 & 2,19 & $-0,06$ & 2,25 \\
\hline Calf_Left & 5,67 & 6,90 & 4,73 & 2,17 \\
\hline Calf_Right & 1,84 & 3,42 & 0,69 & 2,73 \\
\hline Calf_Height_Left & 0,78 & 2,52 & $-0,76$ & 3,28 \\
\hline Calf_Height_Right & 1,39 & 3,36 & $-0,11$ & 3,46 \\
\hline Outseam_Left & $-0,11$ & 0,07 & $-0,30$ & 0,37 \\
\hline Outseam_Right & 0,14 & 0,41 & $-0,11$ & 0,51 \\
\hline Inseam_Left & 0,04 & 0,70 & $-0,49$ & 1,19 \\
\hline Inseam_Right & 0,12 & 0,77 & $-0,31$ & 1,09 \\
\hline Thigh_95_Left & 3,68 & 5,24 & 1,85 & 3,39 \\
\hline Thigh_95_Right & 2,02 & 4,34 & 0,46 & 3,88 \\
\hline Thigh_90_Left & 3,28 & 5,13 & 2,08 & 3,05 \\
\hline Thigh_90_Right & 1,60 & 3,59 & 0,06 & 3,53 \\
\hline Thigh_85_Left & 3,50 & 5,22 & 2,35 & 2,87 \\
\hline Thigh_85_Right & 1,83 & 3,53 & 0,71 & 2,82 \\
\hline Thigh_80_Left & 3,38 & 5,72 & 2,04 & 3,68 \\
\hline Thigh_80_Right & 1,92 & 3,87 & 0,35 & 3,53 \\
\hline Thigh_75_Left & 3,28 & 5,85 & 1,19 & 4,66 \\
\hline Thigh_75_Right & 1,65 & 3,64 & 0,22 & 3,42 \\
\hline Thigh_70_Left & 3,71 & 5,45 & 1,60 & 3,85 \\
\hline Thigh_70_Right & 1,90 & 3,28 & 0,31 & 2,97 \\
\hline Thigh_65_Left & 4,91 & 6,72 & 3,06 & 3,66 \\
\hline Thigh_65_Right & 2,41 & 3,76 & 1,08 & 2,68 \\
\hline Thigh_60_Left & 5,09 & 6,97 & 3,52 & 3,46 \\
\hline Thigh_60_Right & 2,17 & 4,33 & 0,87 & 3,46 \\
\hline Thigh_55_Left & 4,96 & 5,99 & 4,01 & 1,98 \\
\hline Thigh_55_Right & 1,93 & 3,04 & 0,94 & 2,10 \\
\hline Thigh_50_Left & 5,44 & 6,31 & 4,67 & 1,64 \\
\hline Thigh_50_Right & 1,97 & 3,50 & 0,62 & 2,88 \\
\hline Average difference & 2,10 & & & \\
\hline
\end{tabular}

Left side average difference from NX16\%

Right side average difference from NX16\% 1,90

Difference between sides from NX16\%

2,46 


\subsubsection{The crotch point}

It is hard to get information about how different body scanners are detecting the crotch point landmark. From the literature several definitions are found:

- a set of three-dimensional body scanned data is projected onto the coronal plane, locally maximum and minimum points on the silhouette curves can be used to extract crotch point [6];

- point calculated midway between the right and left trochanterion landmarks at the level of crotch height as measured with the anthropometer [7].
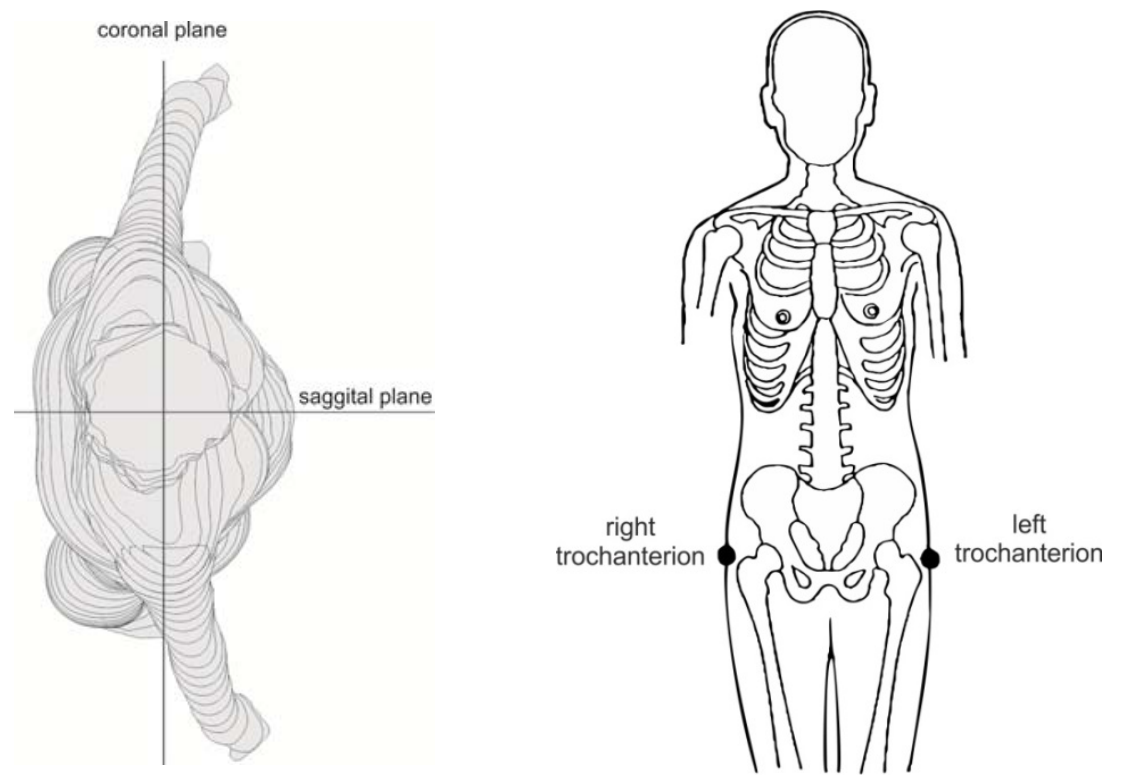

Fig. 3. Crotch point

This question has been asked from $\mathrm{Tc}^{2}$ support and they do not disclose the details about crotch point landmark detection, because it is Intellectual property.

Still, I can find half explanation in $\mathrm{Tc}^{2}$ MEP Editor Tutorial:

Landmarks Select extracts a list of 9 landmarks located on the body and writes them out to a text file. The number of landmarks extracted is displayed as the value of the measurement.

The output text file is written in the .ord directory with the name of the subject as the file name and landmarks as the file extension. The xyz values all reference a point on the floor directly below the crotch point.

This is a Landmarks file opened with Excel. The columns are labeled with Front, Left, and Up meaning $x, y$, and $z$.

The landmarks in the picture are circled with red for clarity. They are not normally displayed this way on the computer monitor.

\begin{tabular}{|l|l|l|l|}
\hline Units = centimeters & Front & Left & Up \\
\hline \hline CrotchPoint & 0.0 & -0.0 & 68.7 \\
\hline LeftArmpit & 7.6 & 15.5 & 121.9 \\
\hline RightArmpit & 4.8 & -16.2 & 121.8 \\
\hline BackNeck & -9.8 & 1.7 & 138.2 \\
\hline LeftSideNeckPoint & -3.1 & 11.6 & 137.2 \\
\hline RightSideNeckPoint & -3.3 & -8.1 & 137.7 \\
\hline FrontCenterNeckPoint & 4.1 & 1.7 & 132.5 \\
\hline LeftShoulderPoint & -1.7 & 20.7 & 131.0 \\
\hline RightShoulderPoint & -0.3 & -18.5 & 133.0 \\
\hline
\end{tabular}

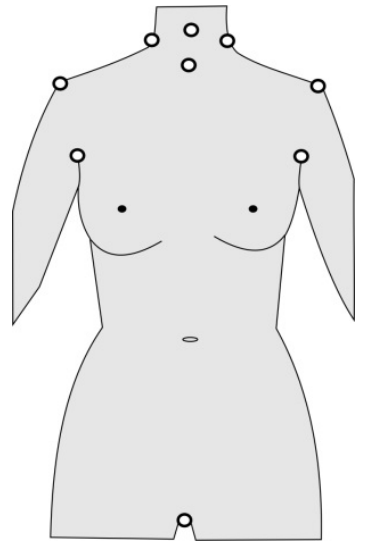

Fig. 4. Landmark presentation in excel and in body image. 
Definition of the $\mathrm{TC}^{2}$ does not say how it can be found from widthwise (on the line from the front waist point to the back waist point) on the horizontal level of crotch (see Fig. 5.Crotch level on the horizontal level).

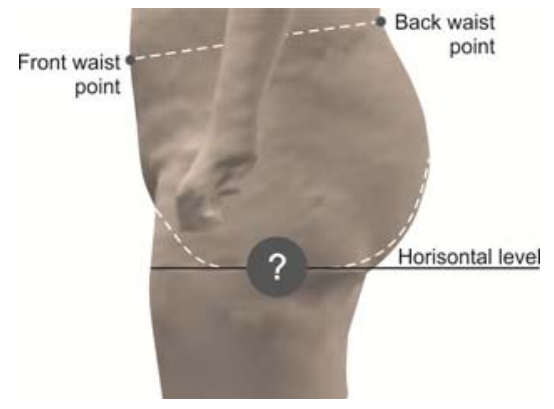

Fig. 5. Crotch level on the horizontal level

When the crotch landmark is extracted with scanner measurement extraction profile it is expected to be in the same place in person's different scans which are taken consequently (body scanner can be set to take three scans in a row from one person).

In reality, the crotch point is fluctuating (see Fig. 6 . Crotch point fluctuation in different scans), and this is causing balance problems in garment fitting, and this can cause even remake of the whole garment.

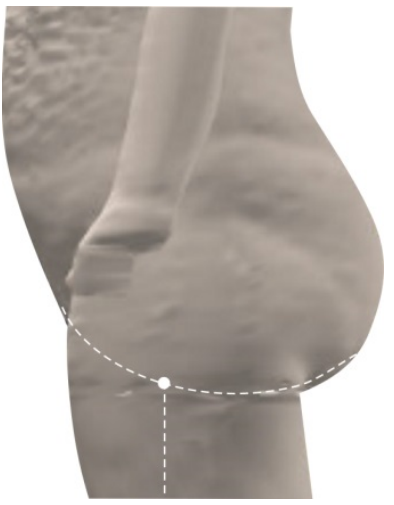

Person 1 in the first scan

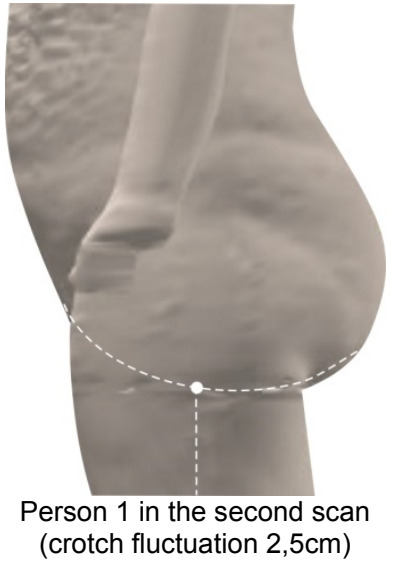

Fig. 6. Crotch point fluctuation in different scans

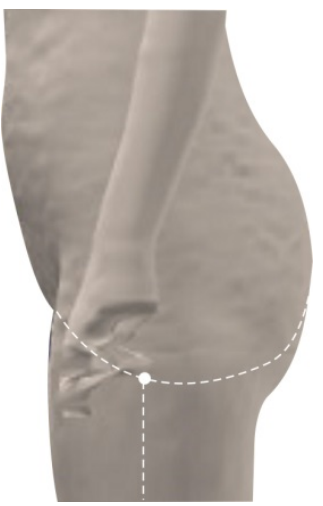

Person 2 in the first scan

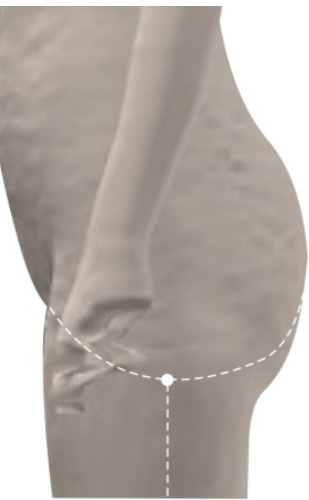

Person 2 in the second scan (crotch fluctuation $2,0 \mathrm{~cm})$

The crotch point should be placed in side view on horizontal level proportionally in the same place for different persons, but the placement is very different depending on the landmark definition. See Fig. 7. Crotch point position in different people.
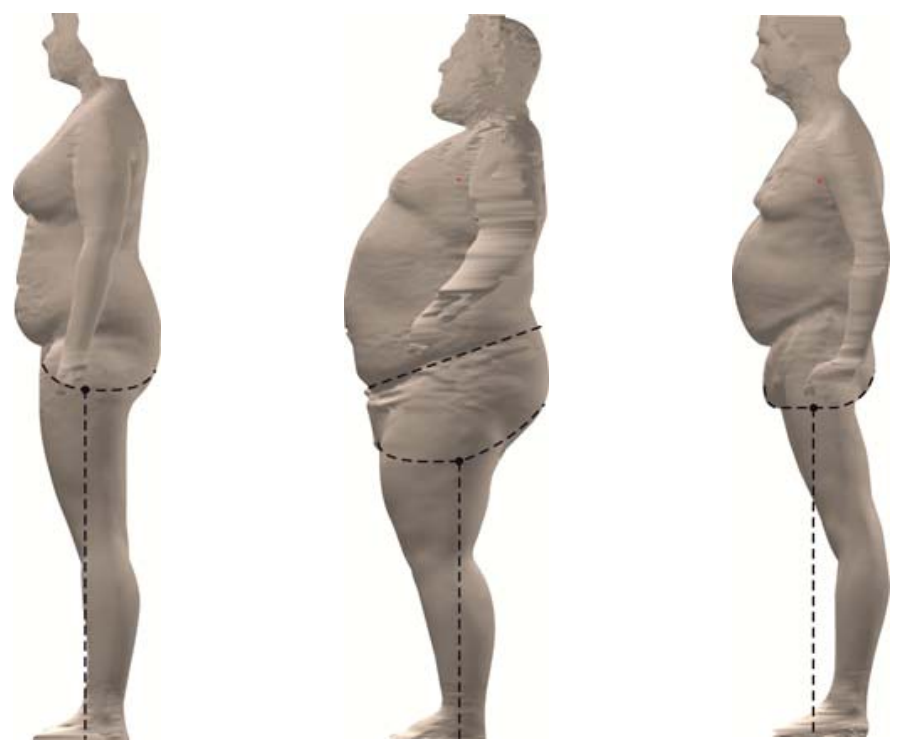

Fig. 7. Crotch point position in different people. 
In clothing construction the crotch includes the area of a pair of trousers or shorts where the legs join together. The bottom of the crotch defines one end of the inseam [8], The crotch point in pattern is defined in figure 8.

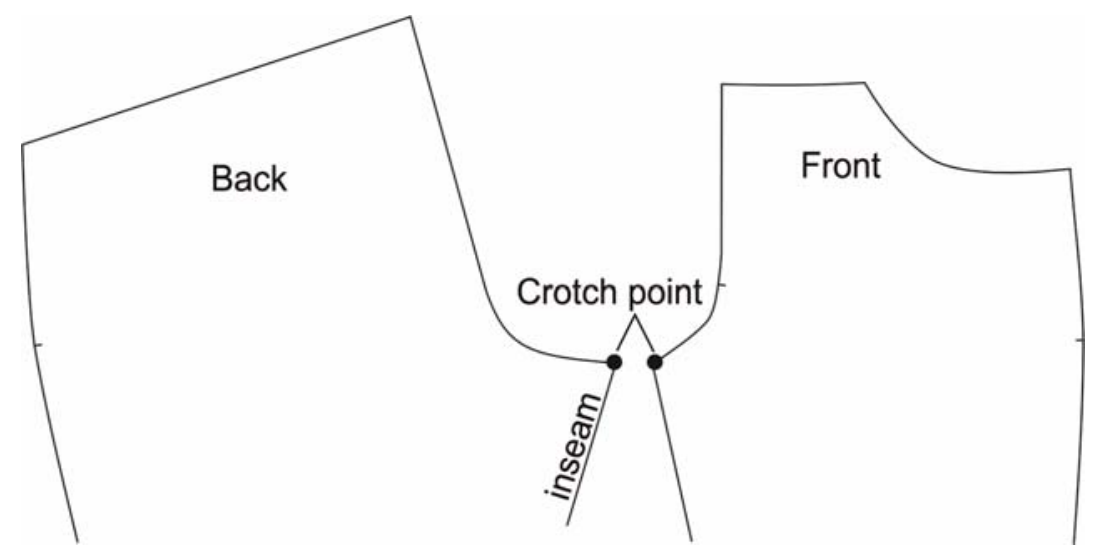

Fig. 8. Crotch point in trousers

To produce fitting garments, the crotch point in the horizontal level cannot fluctuate and should be in relation with middle point of the legs which would be the center point of front thigh and back thigh. See Fig. 9. Recommended crotch point.
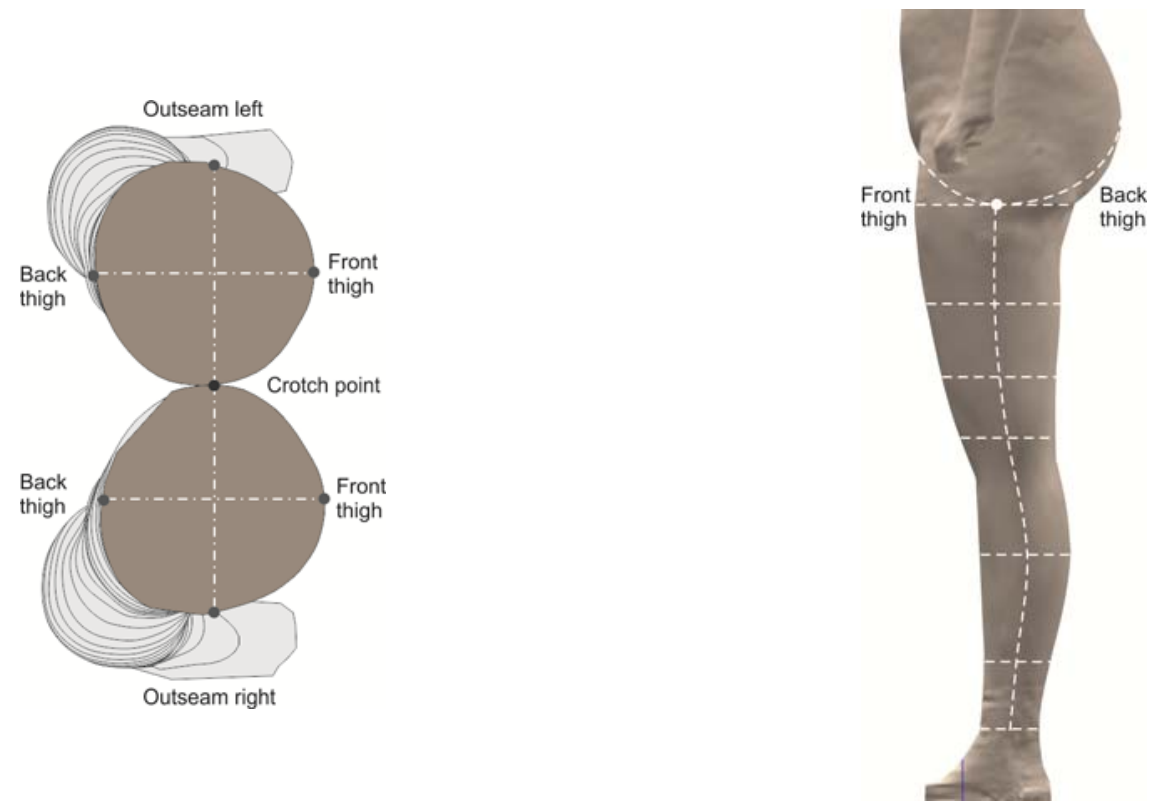

Fig. 9. Recommended crotch point

\section{Conclusions}

First of all -body scanners of different companies' should be compared against each other.

To obtain precision of measurements made with different companies' body scanners, we need to develop "standard mannequin" which would allow us to control the accuracy of the body scanners and the measurement extraction profiles. It is not acceptable, that the body scanners in the market are not ready for the customer and are producing errors in extracting the body measurements, defining body landmarks randomly even for one person, and body measurements of the test group persons are fluctuating between two different scanners of the same company. The new body scanners in the market need to be controlled by accreditation institute who would give certificates if they would correspond to the standard. 
Crotch point landmark detection methods should be made public, so that companies could fix any issues concerning crotch point.

Inseam placement on the horizontal level, also the front crotch and back crotch starting point should be the middle point of the legs and this point would be the center point of front thigh and back thigh.

\section{References}

[1] G. Stylios, J. Fan, J. Sotomi ja R. Deavon, "A new concept in garment manufacture," International Journal of Clothing Science and Technology, osa/vuosik. 4(5), pp. 45-48, 1992.

[2] S. Petrak ja D. Rogale, "Methods of automatic computerized cutting pattern construction.," International Journal of Clothing Science, osa/vuosik. 13, nro 3/4, pp. 229-239, 2001.

[3] T. K. S. Kang, "Development of three-dimensional apparel CAD system. Part I: Flat garment pattern drafting system.," International Journal of Clothing Science and Technology, osa/vuosik. 12, nro 1, pp. 26-38, 2000.

[4] S. Petrak ja D. Rogale, "Systematic representation and application of a 3D computer-aided garment construction method (Part," International Journal of Clothing Science and Technology, osa/vuosik. 18, nro 3, pp. 179-187, 2006.

[5] Naisten Vaatetuksen Mittataulukko N-2001, Helsinki: Tekstiili- ja vaatetusteollisuus ry, 2001.

[6] J.-M. Lu ja M.-J. Wang, "Automated landmark extraction from three-dimensional Whole body scanned data". United States Patentti US 7,561,726 B2, 14 July 2009.

[7] S. Blackwel, K. Robinette, H. Daanen, M. Boehmer, S. Fleming, S. Kelly, T. Brill, D. Hoeferlin ja D. Burnsides, "Civilian American and European surface anthropometry resource (CAESAR)," Warrendale, PA..

[8] 28. 09. 2013. [Online]. Available: http://en.wikipedia.org/wiki/Crotch. 